\title{
FILTRATIONS OF RATIONAL REPRESENTATIONS OF REDUCTIVE GROUPS OF SEMISIMPLE RANK 1
}

\author{
STEPHEN DOTY
}

(Communicated by Warren J. Wong)

\begin{abstract}
A detailed study is made of the affine coordinate ring of the Chevalley group $\mathrm{SL}_{2}$ over the integers as base ring. Certain applications to the representation theory of groups of semisimple rank 1 are made, including the construction of a filtration on modules obtained by inducing a character of a maximal torus $T$ from $T$ up to the group. We show this filtration extends the Jantzen-Andersen filtration on the dual Weyl module with highest weight given by that character, in case the character in question is dominant.
\end{abstract}

\section{INTRODUCTION}

In [4], J. C. Jantzen (see also [5, 6]) constructed a certain canonical filtration on Weyl modules for a semisimple algebraic group $G$ over an algebraically closed field of nonzero characteristic, using the existence of a contravariant form. The Weyl modules appear as the top nonvanishing degree of sheaf cohomology of homogeneous line bundles on $G / B, B$ a Borel subgroup of $G$. In [1], H. H. Andersen (see also [2]) extended Jantzen's filtrations to the other degrees of cohomology, and showed that if the filtration levels are respected by certain intertwining mappings, then G. Lusztig's conjectured formula [7] for the character of a simple module for $G$ would follow.

We are interested in extending Andersen's method to filter modules of the form $\operatorname{ind}_{T}^{G} \lambda$ for $\lambda$ a character on a maximal torus $T$ in $G$. In this article we show that such an extension can be made in the case where $G$ is a connected reductive group of semisimple rank 1. In a companion article [3] we use the results of this article to study the same question for a general reductive group. Once the filtration on $\operatorname{ind}_{T}^{G} \lambda$ has been constructed it is fairly easy to get a corresponding filtration on the so-called "baby Verma module" $Z_{r}(\lambda)$, which should be the analogue of Jantzen's filtration of the Weyl modules. Obtaining such a filtration was a primary motivation for this work. We do not pursue that application here as it is considered in the general case in [3].

By standard arguments, the situation for a group of semisimple rank 1 reduces to $\mathrm{SL}_{2}$, so we only consider that case explicitly. The notation is standard.

Received by the editors January 31, 1989 and, in revised form, June 6, 1989.

1980 Mathematics Subject Classification (1985 Revision). Primary 20G05, 20G15, 20 G40. 
Background and definitions may be found in [6].

Let $\alpha$ be the lone positive root, where $B$ corresponds to $-\alpha$. If $\lambda$ is a character on $T$, we identify $\lambda$ with the integer $\left\langle\alpha^{\vee}, \lambda\right\rangle$. In $\S 1$ we show that for each dominant $\lambda$ there is an embedding

$$
H_{\mathbf{Z}}^{0}(\lambda)=\operatorname{ind}_{B_{\mathbf{Z}}}^{G_{\mathbf{Z}}} \lambda \rightarrow \operatorname{ind}_{T_{\mathbf{Z}}}^{G_{\mathbf{Z}}}(\lambda)
$$

and for each positive integer $q$ such that $\lambda \geq q-1$ an embedding (later $q$ is specialized to a power of $p$ )

$$
\begin{aligned}
H_{\mathbf{Z}}^{1}\left(s_{\alpha} \cdot \lambda\right) & =H_{\mathbf{Z}}^{1}(-\lambda-2)=R^{1} \operatorname{ind}_{B_{\mathbf{Z}}}^{G_{\mathbf{Z}}}(-\lambda-2) \rightarrow \operatorname{ind}_{T_{\mathbf{Z}}}^{G_{\mathbf{Z}}}(2 q-2-\lambda) \\
& =\operatorname{ind}_{T_{\mathbf{Z}}}^{G_{\mathbf{Z}}}\left(s_{\alpha} \cdot \lambda-q s_{\alpha} \cdot 0\right) .
\end{aligned}
$$

These embeddings are unique up to scalar. We show in $\S 2$ that, for $\lambda \geq q-1$, there are $G_{\mathbf{Z}}$ maps

$$
\operatorname{ind}_{T_{\mathbf{z}}}^{G_{\mathbf{z}}}(2 q-2-\lambda) \rightarrow \operatorname{ind}_{T_{\mathbf{z}}}^{G_{\mathbf{z}}} \lambda
$$

and

$$
\operatorname{ind}_{T_{\mathbf{z}}}^{G_{\mathbf{z}}} \lambda \rightarrow \operatorname{ind}_{T_{\mathbf{z}}}^{G_{\mathbf{z}}}(2 q-2-\lambda)
$$

which coincide with the maps used by Andersen [1] when restricted to the image of the above embeddings. It is these maps which give us, by the method of Andersen, the filtrations. In $\S 3$, we show that our filtration on $\operatorname{ind}_{T_{\mathrm{z}}}^{G_{\mathrm{z}}} \lambda$ coincides with Andersen's when restricted to $H_{Z}^{0}(\lambda)$. The proof of that result is nontrivial, (even for $\mathrm{SL}_{2}$ ), requiring the detailed information on the embedding of $H_{\mathbf{Z}}^{1}(-\lambda-2)$ in $\operatorname{ind}_{T_{\mathbf{Z}}}^{G_{\mathbf{Z}}}(2 q-2-\lambda)$ provided by Proposition 1 at the end of $\S 1$.

\section{EMBEDDINGS IN THE COORDINATE RING}

Let $G_{\mathbf{Z}}=\left(\mathrm{SL}_{2}\right)_{\mathbf{Z}}$ be the affine algebraic $\mathrm{Z}$-group scheme given for any commutative ring $A$ by $G_{\mathbf{Z}}(A)=$ all $2 \times 2$ matrices over $A$ of determinant 1 . The affine coordinate ring $\mathbf{Z}\left[G_{\mathbf{Z}}\right]$ is the quotient of the polynomial algebra $\mathbf{Z}\left[X_{11}, X_{12}, X_{21}, X_{22}\right]$ by the ideal $\left(X_{11} X_{22}-X_{12} X_{21}-1\right)$. Write $x_{i j}$ for the image of $X_{i j}$, and identify $x_{i j}$ with the morphism from $G_{\mathrm{Z}}$ to affine one space $\mathbf{A}^{1}$ which maps $g \in G_{\mathbf{Z}}(A)$ onto its $(i, j)$ th entry, for each commutative ring $A$. Let $T_{\mathrm{Z}}$ be the maximal torus in $G_{\mathrm{Z}}$ given by $T_{\mathrm{Z}}(A)=$ diagonal matrices in $G_{\mathbf{Z}}(A)$, and $B_{\mathbf{Z}}$ (resp., $B_{\mathbf{Z}}^{+}$) the Borel subgroup given by $B_{\mathbf{Z}}(A)=$ lower triangular matrices in $G_{\mathbf{Z}}(A)$, (resp., $B_{\mathbf{Z}}^{+}(A)=$ upper triangular matrices in $G_{\mathbf{Z}}(A)$ ). Let $\varepsilon_{1}$ be the character on $T_{\mathrm{Z}}$ given by restricting $x_{11}$ to $T_{\mathrm{Z}}$. Then $\alpha=2 \varepsilon_{1}$ is the positive root (determined by $B_{\mathrm{Z}}^{+}$), $-\alpha=-2 \varepsilon_{1}$ is the negative root (determined by $\left.B_{\mathrm{Z}}\right)$. Since $X\left(T_{\mathrm{Z}}\right)=\mathrm{Z} \varepsilon_{1}$, we shall from now on identify $\varepsilon_{1}$ with the unit in $\mathbf{Z}$, so that characters $\lambda \in X\left(T_{\mathbf{Z}}\right)$ are identified with integers.

A typical element of $\mathbf{Z}\left[G_{\mathbf{Z}}\right]$ may be expressed as a Z-linear combination of monomials $x^{\beta}=x_{11}^{\beta_{11}} x_{12}^{\beta_{12}} x_{21}^{\beta_{21}} x_{22}^{\beta_{22}}$ for various nonnegative integers $\beta_{11}, \beta_{12}$, 
$\beta_{21}, \beta_{22}$. But the set of all monomials is not a basis for the coordinate ring as we have, for all $i \geq 0$,

$$
x_{12}^{i} x_{21}^{i}=\sum_{j=0}^{i}(-1)^{i-j}\left(\begin{array}{l}
i \\
j
\end{array}\right) x_{11}^{j} x_{22}^{j} .
$$

There is a $\mathbf{Z}$-basis of $\mathbf{Z}\left[G_{\mathbf{Z}}\right]$ consisting of monomials: just take the set of all monomials $x^{\beta}$ for which $\beta_{12}$ or $\beta_{21}$ is zero.

Now $\mathbf{Z}\left[G_{\mathbf{Z}}\right]$ is a module for $G_{\mathbf{Z}}$ in two different ways, via the right and left regular representations. For the right regular representation, any $g \in \mathrm{SL}_{2}(A)$ will act on $\mathrm{Z}\left[G_{\mathbf{Z}}\right] \otimes A$ by the rule (we are going to be notationally sloppy throughout and identify $x^{\beta}$ with $\left.x^{\beta} \otimes 1\right)$

$$
\begin{aligned}
g \cdot x^{\beta} & =\prod_{i, j}\left(\sum_{k} x_{i k} g_{k j}\right)^{\beta_{i j}} \\
& =\sum_{\beta=\beta(1)+\beta(2)}\left(\prod \frac{\beta_{i j} !}{\beta_{i j}(1) ! \beta_{i j}(2) !}\right)\left(\prod x_{i j}^{R_{i} \beta(j)}\right)\left(\prod g_{i j}^{C, \beta(i)}\right),
\end{aligned}
$$

where the sum is carried over all partitions $\beta=\beta(1)+\beta(2)$ of the integral matrix $\beta$ into a sum of two nonnegative integral matrices $\beta(1)$ and $\beta(2)$, and where, for a matrix, $R_{i}$ stands for the sum of the entries in row $i$, and $C_{j}$ the sum of entries in column $j$.

For the left regular representation, any $g \in \mathrm{SL}_{2}(A)$ acts on $\mathbf{Z}\left[G_{\mathbf{Z}}\right] \otimes A$ by the rule

$$
\begin{aligned}
g^{-1} \cdot x^{\beta} & =\prod_{i, j}\left(\sum_{k} g_{i k} x_{k j}\right)^{\beta_{i j}} \\
& =\sum_{\beta=\beta(1)+\beta(2)}\left(\prod \frac{\beta_{i j} !}{\beta_{i j}(1) ! \beta_{i j}(2) !}\right)\left(\prod g_{i j}^{R_{i} \beta(j)}\right)\left(\prod x_{i j}^{C, \beta(i)}\right) .
\end{aligned}
$$

In particular, the right $T_{\mathrm{z}}$-weight of $x^{\beta}$ is $C_{1} \beta-C_{2} \beta=\left(\beta_{11}+\beta_{21}\right)-\left(\beta_{12}+\right.$ $\left.\beta_{22}\right)$, and the left $T_{\mathrm{Z}}$-weight of $x^{\beta}$ is $R_{2} \beta-R_{1} \beta=\left(\beta_{21}+\beta_{22}\right)-\left(\beta_{11}+\beta_{12}\right)$.

Lemma 1. Let $\lambda \in X\left(T_{\mathbf{Z}}\right)$. The induced $G_{\mathbf{Z}}$ module $\operatorname{ind}_{T_{\mathbf{Z}}}^{G_{\mathbf{Z}}} \lambda$ equals $\mathrm{Z}\left[G_{\mathbf{Z}}\right]_{-\lambda}$, the $-\lambda$ weight space of $\mathbf{Z}\left[G_{\mathbf{Z}}\right]$ under the right regular action of $T_{\mathbf{Z}}$.

Proof. This is a general fact which is immediate from the definition of induction (cf. [6, I.3.3]).

Thus, we see that $\operatorname{ind}_{T_{\mathrm{Z}}}^{G_{\mathrm{Z}}} \lambda$ equals the Z-span of all monomials $x^{\beta}$ for which $-\lambda=\left(\beta_{11}+\beta_{21}\right)-\left(\beta_{12}+\beta_{22}\right)$.

Lemma 2. Suppose $\lambda \geq 0$. The induced $G_{\mathrm{Z}}$ module $H_{\mathbf{Z}}^{0}(\lambda)=\operatorname{ind}_{B_{Z}}^{G_{Z}} \lambda$ embeds canonically in $\operatorname{ind}_{T_{\mathbf{Z}}}^{G_{\mathbf{Z}}} \lambda$. $H_{\mathbf{Z}}^{0}(\lambda)$ has a $\mathbf{Z}$-basis of monomials

$$
\left\{v_{i}=x_{12}^{i} x_{22}^{\lambda-i}\right\}_{i=0}^{\lambda}
$$


Proof. By definition, $H_{\mathbf{Z}}^{0}(\lambda)=\mathbf{Z}\left[G_{\mathbf{Z}}\right]_{-\lambda} \cap \mathbf{Z}\left[G_{\mathbf{Z}}\right]^{U_{\mathbf{Z}}}$, where $U_{\mathbf{Z}}$ is the unipotent radical of $B_{\mathrm{Z}}$. Here the $U_{\mathrm{Z}}$ invariant points are computed relative to the right regular representation. One checks that $\mathbf{Z}\left[G_{\mathbf{Z}}\right]^{U_{\mathbf{Z}}}=\sum_{i, j} \mathbf{Z} x_{12}^{i} x_{22}^{j}$ and the result follows.

Let $q$ be a positive integer.

Lemma 3. Suppose $\lambda \geq q-1$. There is an embedding of $H_{\mathbf{Z}}^{1}(-\lambda-2)=$ $R^{1} \operatorname{ind}_{B_{\mathbf{Z}}}^{G_{Z}}(-\lambda-2)$ in $\operatorname{ind}_{T_{\mathbf{Z}}}^{G_{\mathbf{Z}}}(2 q-2-\lambda)$. The embedding is unique up to scalar multiple, and can be so chosen that the image of $H_{\mathbf{Z}}^{1}(-\lambda-2)$ in $\operatorname{ind}_{T_{\mathbf{Z}}}^{G_{Z}}(2 q-2-\lambda)$ has the $\mathbf{Z}$-basis

$$
\left\{v_{i}^{\prime}=\sum_{a+b=i}\left(\begin{array}{c}
\lambda-(q-1) \\
a
\end{array}\right)\left(\begin{array}{c}
q-1 \\
b
\end{array}\right) x_{11}^{a} x_{12}^{b} x_{21}^{\lambda-(q-1)-a} x_{22}^{q-1-b}\right\}_{i=0}^{\lambda} .
$$

Proof. First note that the $\left\{v_{0}^{\prime}, v_{1}^{\prime}, \ldots, v_{\lambda}^{\prime}\right\}$ are linearly independent because each $v_{i}^{\prime}$ has left $T_{\mathrm{Z}}$ weight $\lambda-2 i$ and so the $v_{i}^{\prime}$ all belong to distinct weight spaces. Comparing with [6, II.5.2(d)] we see that it is sufficient to show the standard root subgroups act by

$$
\begin{gathered}
{\left[\begin{array}{ll}
1 & z \\
0 & 1
\end{array}\right] \cdot v_{i}^{\prime}=\sum_{j=0}^{i}\left(\begin{array}{c}
\lambda-j \\
\lambda-i
\end{array}\right) z^{i-j} v_{j}^{\prime},} \\
{\left[\begin{array}{ll}
1 & 0 \\
z & 1
\end{array}\right] \cdot v_{i}^{\prime}=\sum_{j=i}^{\lambda}\left(\begin{array}{l}
j \\
i
\end{array}\right) z^{j-i} v_{j}^{\prime},}
\end{gathered}
$$

for all $z \in A$, for all $A$, and for all $i \quad(0 \leq i \leq \lambda)$. We verify (1.7) below. First we have from the definition of $v_{i}^{\prime}$ that

$$
\begin{aligned}
{\left[\begin{array}{ll}
1 & 0 \\
z & 1
\end{array}\right] \cdot v_{i}^{\prime} } & (-1)^{i} \sum_{a+b=i}\left(\begin{array}{c}
\lambda-(q-1) \\
a
\end{array}\right)\left(\begin{array}{c}
q-1 \\
b
\end{array}\right) x_{11}^{a} x_{12}^{b}\left(-z x_{11}+x_{21}\right)^{\lambda-(q-1)-a} \\
& \times\left(-z x_{12}+x_{22}\right)^{q-1-b} \\
= & \sum_{j=i}^{\lambda} z^{j-i}(-1)^{j-i} \sum_{\substack{a+b=i \\
c+d=j-i}} C_{\lambda, q, a, b, c, d} x_{11}^{a+c} x_{12}^{b+d} x_{21}^{\lambda-(q-1)-(a+c)} x_{22}^{q-1-(b+d)},
\end{aligned}
$$

where

$$
\begin{aligned}
C_{\lambda, q, a, b, c, d} & =\left(\begin{array}{c}
\lambda-(q-1) \\
a
\end{array}\right)\left(\begin{array}{c}
q-1 \\
b
\end{array}\right)\left(\begin{array}{c}
\lambda-(q-1)-a \\
c
\end{array}\right)\left(\begin{array}{c}
q-1-b \\
d
\end{array}\right) \\
& =\left(\begin{array}{c}
\lambda-(q-1) \\
a+c
\end{array}\right)\left(\begin{array}{c}
a+c \\
a
\end{array}\right)\left(\begin{array}{c}
q-1 \\
b+d
\end{array}\right)\left(\begin{array}{c}
b+d \\
b
\end{array}\right) .
\end{aligned}
$$


Hence,

$$
\begin{gathered}
{\left[\begin{array}{ll}
1 & 0 \\
z & 1
\end{array}\right] \cdot v_{i}^{\prime}=\sum_{j=i}^{i} z^{j-i}(-1)^{j-i} \sum_{\substack{u+v=j \\
a+b=i}}\left(\begin{array}{c}
\lambda-(q-1) \\
u
\end{array}\right)\left(\begin{array}{c}
q-1 \\
v
\end{array}\right)\left(\begin{array}{l}
u \\
a
\end{array}\right)\left(\begin{array}{l}
v \\
b
\end{array}\right)} \\
\times x_{11}^{u} x_{12}^{\prime \prime} x_{21}^{\lambda-(q-1)-u} x_{22}^{q-1-v} \\
=\sum_{j=i}^{\lambda} z^{j-i}(-1)^{j-i} \sum_{u+v=j}\left(\begin{array}{c}
\lambda-(q-1) \\
u
\end{array}\right)\left(\begin{array}{c}
q-1 \\
v
\end{array}\right) \\
\times \sum_{a=0}^{i}\left(\begin{array}{c}
u \\
a
\end{array}\right)\left(\begin{array}{c}
v \\
i-a
\end{array}\right) x_{11}^{u} x_{12}^{v} x_{21}^{i-(q-1)-u} x_{22}^{q-1-v} .
\end{gathered}
$$

Thus, (1.7) will follow if we can show that

$$
\left(\begin{array}{l}
j \\
i
\end{array}\right)=\sum_{a=0}^{i}\left(\begin{array}{l}
u \\
a
\end{array}\right)\left(\begin{array}{l}
j-u \\
i-a
\end{array}\right) \quad \text { for all } i, j, \text { and } u \quad(0 \leq u \leq j)
$$

This is a simple exercise. One way to see it is to expand $(1+x)^{j},(1+x)^{u}$, and $(1+x)^{j-u}$ by the binomial formula and then multiply; the result $(1.8)$ is obtained by comparing coefficients. Thus, (1.7) is valid. We leave the verification of (1.6), which is similar, to the reader.

Finally, to see that the embedding is unique up to scalar, note that $H_{\mathrm{Z}}^{1}(-\lambda-2)$ has a unique (up to scalar) maximal vector, which is, by definition, a nonzero element of the $U_{\mathbf{Z}}^{+}$invariant points in $H_{\mathbf{Z}}^{1}(-\lambda-2)$. Here $U_{\mathbf{Z}}^{+}$is the unipotent radical of $B_{\mathbf{Z}}^{+}$. Now any embedding of $G_{\mathbf{Z}}$ modules $H_{\mathbf{Z}}^{1}(-\lambda-2) \rightarrow$ ind $_{T_{Z}}^{G_{Z}}(2 q-2-\lambda)$ must map this maximal vector to a $U_{Z}^{+}$invariant point in ind $_{T_{z}}^{G_{z}}(2 q-2-\lambda)$. It is easy to verify that $\mathbf{Z}\left[G_{\mathbf{Z}}\right]^{U_{Z}^{+}}=\sum_{i, j} \mathbf{Z} x_{21}^{i} x_{22}^{j}$. (Of course, $U_{\mathrm{Z}}^{+}$is acting via the left regular representation here.) Thus, it follows that $\mathbf{Z}\left[G_{\mathbf{Z}}\right]_{(2 q-2-\lambda)} \cap \mathbf{Z}\left[G_{\mathbf{Z}}\right]^{l_{Z}^{+}}=\mathbf{Z} x_{21}^{\lambda-(q-1)} x_{22}^{q-1}$. Noting that $v_{0}^{\prime}=x_{21}^{\lambda-(q-1)} x_{22}^{q-1}$, the uniqueness statement follows, and the proof of Lemma 3 is complete.

Remarks. 1. As the reader has probably already realized, the basis (1.5) was derived from applying the element $(-1)^{i} X_{-r . i}$ of the Kostant Z-form (see [6, II.2.16]), to the maximal vector $v_{0}^{\prime}$.

2. Another description of the element $v_{i}^{\prime}$ is the following: $v_{i}^{\prime}$ is the coefficient of $t^{i}$ in the polynomial $\left(x_{11}+t x_{21}\right)^{\lambda-(q-1)}\left(x_{12}+t x_{22}\right)^{q-1}$. 
Lemma 4. Assume $\lambda \geq q-1$. In terms of the basis $\left\{x^{\beta}: \beta_{12} \beta_{21}=0\right\}_{\beta}$ of the coordinate ring $\mathbf{Z}\left[G_{\mathbf{Z}}\right]$, we have

$$
v_{i}^{\prime}=\left\{\begin{array}{cc}
\sum_{j=0}^{i}(-1)^{j}\left(\begin{array}{c}
\lambda-j \\
i-j
\end{array}\right)\left(\begin{array}{c}
q-1 \\
j
\end{array}\right) x_{11}^{i-j} x_{21}^{\lambda-(q-1)-i} x_{22}^{(q-1)-j}, & \text { if } i \leq \lambda-(q-1), \\
\sum_{j=0}^{\lambda-(q-1)}(-1)^{j}\left(\begin{array}{c}
\lambda-j \\
i
\end{array}\right)\left(\begin{array}{c}
\lambda-(q-1) \\
j
\end{array}\right) x_{11}^{\lambda-(q-1)-j} x_{12}^{i-(\lambda-(q-1))} x_{22}^{\lambda-j-i}, & \text { if } i \geq \lambda-(q-1) .
\end{array}\right.
$$

Remark. The two formulas in (1.9) do coincide when $i=\lambda-(q-1)$, for in that case we have for all $0 \leq j \leq \lambda-(q-1)$ that

$$
\begin{aligned}
\left(\begin{array}{c}
\lambda-j \\
\lambda-(q-1)-j
\end{array}\right)\left(\begin{array}{c}
q-1 \\
j
\end{array}\right) & =\left(\begin{array}{c}
\lambda-j \\
q-1
\end{array}\right)\left(\begin{array}{c}
q-1 \\
j
\end{array}\right) \\
& =\left(\begin{array}{c}
\lambda-j \\
\lambda-(q-1)
\end{array}\right)\left(\begin{array}{c}
\lambda-(q-1) \\
j
\end{array}\right) .
\end{aligned}
$$

Proof of Lemma 4. By (1.5) we have

$$
v_{i}^{\prime}=\sum_{b=0}^{i}\left(\begin{array}{c}
\lambda-(q-1) \\
i-b
\end{array}\right)\left(\begin{array}{c}
q-1 \\
b
\end{array}\right) x_{11}^{i-b} x_{12}^{b} x_{21}^{\lambda-(q-1)-i+b} x_{22}^{(q-1)-b}
$$

Assume that $0 \leq i \leq \lambda-(q-1)$. Then, applying the relation $x_{12}^{b} x_{21}^{b}=$ $\sum_{j=0}^{b}(-1)^{j}\left(\begin{array}{l}b \\ j\end{array}\right) x_{11}^{b-j} x_{22}^{b-j}$, formula (1.10) takes the form

$$
\begin{aligned}
v_{i}^{\prime}= & \sum_{b=0}^{i} \sum_{j=0}^{b}(-1)^{j}\left(\begin{array}{c}
\lambda-(q-1) \\
i-b
\end{array}\right)\left(\begin{array}{c}
q-1 \\
b
\end{array}\right)\left(\begin{array}{l}
b \\
j
\end{array}\right) x_{11}^{i-j} x_{21}^{\lambda-(q-1)-i} x_{22}^{(q-1)-j} \\
= & \sum_{j=0}^{i} \sum_{b=j}^{i}(-1)^{j}\left(\begin{array}{c}
\lambda-(q-1) \\
i-b
\end{array}\right)\left(\begin{array}{c}
q-1 \\
b
\end{array}\right)\left(\begin{array}{l}
b \\
j
\end{array}\right) x_{11}^{i-j} x_{21}^{\lambda-(q-1)-i} x_{22}^{(q-1)-j} \\
= & \sum_{j=0}^{i} \sum_{b=0}^{i-j}(-1)^{j}\left(\begin{array}{c}
\lambda-(q-1) \\
i-j-b
\end{array}\right)\left(\begin{array}{c}
q-1 \\
j
\end{array}\right)\left(\begin{array}{c}
(q-1)-j \\
b
\end{array}\right) \\
= & \sum_{j=0}^{i}(-1)^{j}\left(\begin{array}{c}
i-j \\
\sum_{b=0}^{i-j} x_{21}^{\lambda-(q-1)-i} x_{22}^{(q-1)-j}
\end{array}\left(\begin{array}{c}
\lambda-(q-1) \\
i-j-b
\end{array}\right)\left(\begin{array}{c}
(q-1)-j \\
b
\end{array}\right)\right)\left(\begin{array}{c}
q-1 \\
j
\end{array}\right) \\
\times & \times x_{11}^{i-j} x_{21}^{\lambda-(q-1)-i} x_{22}^{(q-1)-j} .
\end{aligned}
$$

Since, by formula (1.8), $\sum_{b=0}^{i-j}\left(\begin{array}{c}\lambda-(q-1) \\ i-j-b\end{array}\right)\left(\begin{array}{c}(q-1)-j \\ b\end{array}\right)=\left(\begin{array}{c}\lambda-j \\ i-j\end{array}\right)$, the desired result

$$
v_{i}^{\prime}=\sum_{j=0}^{i}\left(\begin{array}{c}
\lambda-j \\
i-j
\end{array}\right)\left(\begin{array}{c}
q-1 \\
j
\end{array}\right) x_{11}^{i-j} x_{21}^{\lambda-(q-1)-i} x_{22}^{(q-1)-j}
$$

follows in the case under consideration. 
Assume that $\lambda-(q-1) \leq i \leq \lambda$. By (1.10) we have

$$
\begin{aligned}
v_{i}^{\prime} & =\sum_{b=0}^{i}\left(\begin{array}{c}
\lambda-(q-1) \\
i-b
\end{array}\right)\left(\begin{array}{c}
q-1 \\
b
\end{array}\right) x_{11}^{i-b} x_{12}^{b} x_{21}^{\lambda-(q-1)-(i-b)} x_{22}^{(q-1)-b} \\
& =\sum_{b=i-(\lambda-(q-1))}^{i}\left(\begin{array}{c}
\lambda-(q-1) \\
i-b
\end{array}\right)\left(\begin{array}{c}
q-1 \\
b
\end{array}\right) x_{11}^{i-b} x_{12}^{b} x_{21}^{\lambda-(q-1)-(i-b)} x_{22}^{(q-1)-b} .
\end{aligned}
$$

Replacing $i-b$ by $u$, this takes the form

$$
v_{i}^{\prime}=\sum_{u=0}^{\lambda-(q-1)}\left(\begin{array}{c}
\lambda-(q-1) \\
u
\end{array}\right)\left(\begin{array}{c}
q-1 \\
i-u
\end{array}\right) x_{11}^{u} x_{12}^{i-u} x_{21}^{\lambda-(q-1)-u} x_{22}^{(q-1)-(i-u)} .
$$

Since $i-u \geq \lambda-(q-1)-u$, for all $0 \leq u \leq \lambda-(q-1)$, by applying the relation $\left(x_{12} x_{21}\right)^{\lambda-(q-1)-u}=\sum_{j=0}^{\lambda-(q-1)-u}(-1)^{j}\left(\begin{array}{c}\lambda-(q-1)-u \\ j\end{array}\right)\left(x_{11} x_{22}\right)^{\lambda-(q-1)-u-j}$ to the previous identity, we obtain

$$
\begin{aligned}
& v_{i}^{\prime}=\sum_{u=0}^{\lambda-(q-1)} \sum_{j=0}^{\lambda-(q-1)-u}(-1)^{j}\left(\begin{array}{c}
\lambda-(q-1) \\
u
\end{array}\right)\left(\begin{array}{c}
q-1 \\
i-u
\end{array}\right)\left(\begin{array}{c}
\lambda-(q-1)-u \\
j
\end{array}\right) \\
& \times x_{11}^{\lambda-(q-1)-j} x_{12}^{i-(\lambda-(q-1))} x_{22}^{\lambda-j-i} \\
& =\sum_{j=0}^{\lambda-(q-1)} \sum_{u=0}^{\lambda-(q-1)-j}(-1)^{j}\left(\begin{array}{c}
\lambda-(q-1) \\
u
\end{array}\right)\left(\begin{array}{c}
q-1 \\
i-u
\end{array}\right)\left(\begin{array}{c}
\lambda-(q-1)-u \\
j
\end{array}\right) \\
& \times x_{11}^{\lambda-(q-1)-j} x_{12}^{i-(\lambda-(q-1))} x_{22}^{\lambda-j-i} \\
& =\sum_{j=0}^{\lambda-(q-1)} \sum_{u=0}^{\lambda-(q-1)-j}(-1)^{j}\left(\begin{array}{c}
\lambda-(q-1) \\
j
\end{array}\right)\left(\begin{array}{c}
q-1 \\
i-u
\end{array}\right)\left(\begin{array}{c}
\lambda-(q-1)-j \\
u
\end{array}\right) \\
& \times x_{11}^{\lambda-(q-1)-j} x_{12}^{i-(\lambda-(q-1))} x_{22}^{\lambda-j-i} \\
& =\sum_{j=0}^{\lambda-(q-1)}(-1)^{j}\left(\begin{array}{c}
\lambda-(q-1) \\
j
\end{array}\right)\left(\sum_{u=0}^{\lambda-(q-1)-j}\left(\begin{array}{c}
q-1 \\
i-u
\end{array}\right)\left(\begin{array}{c}
\lambda-(q-1)-j \\
u
\end{array}\right)\right) \\
& \times x_{11}^{\lambda-(q-1)-j} x_{12}^{i-(\lambda-(q-1))} x_{22}^{\lambda-j-i} \text {. }
\end{aligned}
$$

By formula (1.8), since $i \geq \lambda-(q-1)-j$, for all $j$,

$$
\begin{aligned}
\sum_{u=0}^{\lambda-(q-1)-j}\left(\begin{array}{c}
q-1 \\
i-u
\end{array}\right)\left(\begin{array}{c}
\lambda-(q-1)-j \\
u
\end{array}\right) & =\sum_{u=0}^{i}\left(\begin{array}{c}
q-1 \\
i-u
\end{array}\right)\left(\begin{array}{c}
\lambda-(q-1)-j \\
u
\end{array}\right) \\
& =\left(\begin{array}{c}
\lambda-j \\
i
\end{array}\right) .
\end{aligned}
$$

Consequently, the desired formula

$$
v_{i}^{\prime}=\sum_{j=0}^{\lambda-(q-1)}(-1)^{j}\left(\begin{array}{c}
\lambda-(q-1) \\
j
\end{array}\right)\left(\begin{array}{c}
\lambda-j \\
i
\end{array}\right) x_{11}^{\lambda-(q-1)-j} x_{12}^{i-(\lambda-(q-1))} x_{22}^{\lambda-j-i}
$$

follows in this case as well, thus completing the proof. 
The following result is crucial for $\S 3$. Let $p>0$ be an arbitrary prime.

Proposition 1. Let $r$ be a nonnegative integer such that $p^{r}-1 \leq \lambda<p^{r+1}-$ 1. Set $q=p^{r}$. Then, for each $i$ such that $0 \leq i \leq \lambda$, the element $v_{i}^{\prime}$ of $\operatorname{ind}_{T_{\mathrm{Z}}}^{G_{\mathrm{Z}}}(2 q-2-\lambda)$ is not divisible by $p$.

In order to prove this, we shall need the following results.

Let $n$ be a nonnegative integer and let $d_{e}(n)$ be the $e$ th digit in the $p$-adic expansion of $n$ :

$$
n=\sum_{e=0}^{\infty} d_{e}(n) p^{e}, \quad d_{e}(n) \in\{0,1, \ldots, p-1\} .
$$

Lemma 5. Let $\lambda, \mu$ be two nonnegative integers. The binomial coefficient $\left(\begin{array}{l}\lambda \\ \mu\end{array}\right)$ is prime to $p$ if and only if $d_{e}(\lambda) \geq d_{e}(\mu)$, for all $e=0,1,2, \ldots$.

Proof. Check that $\left(\begin{array}{l}\lambda \\ \mu\end{array}\right) \equiv \prod_{e}\left(\begin{array}{l}d_{c}(\lambda) \\ d_{c}(\mu)\end{array}\right)(\bmod p)$.

Corollary. Let $q=p^{r}$. For $0 \leq i \leq q-1,\left(\begin{array}{c}q-1 \\ i\end{array}\right)$ is prime to $p$.

Proof. For each $e=0,1, \ldots, r, d_{e}(q-1)=p-1$.

Proof of Proposition 1.

Case 1. Assume that $0 \leq i<\lambda-(q-1)$. Then, by Lemma 4,

$$
v_{i}^{\prime}=\sum_{j=0}^{i}(-1)^{j}\left(\begin{array}{c}
\lambda-j \\
i-j
\end{array}\right)\left(\begin{array}{c}
q-1 \\
j
\end{array}\right) x_{11}^{i-j} x_{21}^{\lambda-(q-1)-i} x_{22}^{(q-1)-j},
$$

so, by the Corollary, it is enough to exhibit one value of $j$ for which $0 \leq j \leq i$ and $\left(\begin{array}{c}\lambda-j \\ i-j\end{array}\right)$ is prime to $p$. If $i \leq q-1$, then setting $j=i$ does the job. So, assume that $i>q-1$. The integers $\lambda, i$ have the $p$-adic expansions

$$
\lambda=\sum_{e=0}^{r} d_{e}(\lambda) p^{e}, \quad i=\sum_{e=0}^{r} d_{e}(i) p^{e},
$$

where $d_{r}(\lambda)>d_{r}(i)>0$. Set $j=\sum_{e=0}^{r-1} d_{e}(i) p^{e}=i-d_{r}(i) p^{r}$. Then $0 \leq j \leq$ $q-1<i$, and $i-j=d_{r}(i) p^{r}$, so

$$
d_{e}(i-j)= \begin{cases}0, & \text { for } e=0,1, \ldots, r-1, \\ d_{r}(i), & \text { for } e=r .\end{cases}
$$

Hence, for all $e=0, \ldots, r, d_{e}(i-j) \leq d_{e}(\lambda-j)$, and so by Lemma 5 the binomial coefficient $\left(\begin{array}{c}\lambda-j \\ i-j\end{array}\right)$ is prime to $p$.

Case 2. Assume that $\lambda-(q-1) \leq i \leq \lambda$. Then, according to Lemma 4,

$$
v_{i}^{\prime}=\sum_{j=0}^{\lambda-(q-1)}(-1)^{j}\left(\begin{array}{c}
\lambda-j \\
i
\end{array}\right)\left(\begin{array}{c}
\lambda-(q-1) \\
j
\end{array}\right) x_{11}^{\lambda-(q-1)-j} x_{12}^{i-(\lambda-(q-1))} x_{22}^{\lambda-j-i} .
$$

It is convenient to replace $j$ by $\lambda-(q-1)-j$ in the above, so that

$$
\begin{aligned}
v_{i}^{\prime}=\sum_{j=0}^{\lambda-(q-1)}(-1)^{\lambda-(q-1)-j}\left(\begin{array}{c}
(q-1)+j \\
i
\end{array}\right) \\
\times\left(\begin{array}{c}
\lambda-(q-1) \\
j
\end{array}\right) x_{11}^{j} x_{12}^{i-(\lambda-(q-1))} x_{22}^{(q-1)+j-i} .
\end{aligned}
$$


It is enough to exhibit a value of $j$ for which $0 \leq j \leq \lambda-(q-1)$ and $\left(\begin{array}{c}(q-1)+j \\ i\end{array}\right)\left(\begin{array}{c}\lambda-(q-1) \\ j\end{array}\right)$ is prime to $p$. If $i \leq q-1$, then we are finished merely by setting $j=0$, by the corollary again. So, assume $i>q-1$. Then also $\lambda>q-1$, so $\lambda, i$ have $p$-adic expansions

$$
\lambda=\sum_{e=0}^{r} d_{e}(\lambda) p^{e}, \quad i=\sum_{e=0}^{r} d_{e}(i) p^{e}
$$

with $d_{r}(\lambda)>0$ and $d_{r}(i)>0$. Let $E$ be the smallest value of $e$ for which $d_{e}(i)=d_{e}(\lambda) \quad\left(E=r+1\right.$ in case $\left.d_{r}(i) \neq d_{r}(\lambda)\right)$, and set

$$
j=\sum_{e=E-1}^{r} d_{e}(\lambda-(q-1)) p^{e} .
$$

By Lemma 5, $\left(\begin{array}{c}\lambda-(q-1) \\ j\end{array}\right)$ is prime to $p$. Moreover, $d_{E-1}(\lambda)>d_{E-1}(i)$ and one can show that

$$
d_{e}((q-1)+j)= \begin{cases}p-1, & \text { for } e=0, \ldots, E-2, \\ d_{E-1}(\lambda) \text { or } d_{E-1}(\lambda)-1, & \text { for } e=E-1, \\ d_{e}(\lambda), & \text { for } e=E, \ldots, r .\end{cases}
$$

By Lemma $5,\left(\begin{array}{c}(q-1)+j \\ i\end{array}\right)$ is also prime to $p$, and so the proof is complete.

\section{AN ENDOMORPHISM OF THE COORDINATE RING}

From now on regard $\mathrm{Z}\left[G_{\mathbf{Z}}\right]$ as a $G_{\mathbf{Z}}$ module via the left regular representation. Our goal in this section is to construct a $G_{Z}$ module endomorphism of $\mathbf{Z}\left[G_{\mathbf{Z}}\right]$. We do this by constructing the map on each $\operatorname{ind}_{T_{\mathbf{Z}}}^{G_{\mathbf{Z}}} \lambda=\mathbf{Z}\left[G_{\mathbf{Z}}\right]_{-\lambda}$. Throughout this section $q$ denotes a positive integer.

Lemma 6. Suppose $\lambda \geq q-1$. There is a $G_{\mathbf{Z}}$ homomorphism

$$
\phi_{q}(\lambda): \operatorname{ind}_{T_{z}}^{G_{Z}} \lambda \rightarrow \operatorname{ind}_{T_{z}}^{G_{Z}}(2 q-2-\lambda)
$$

such that

$$
\phi_{q}(\lambda)\left(x^{\beta}\right)=(-1)^{\beta_{11}+\beta_{21}} \sum_{c+d=q-1}\left(\begin{array}{c}
\beta_{12} \\
c
\end{array}\right)\left(\begin{array}{c}
\beta_{22} \\
d
\end{array}\right) x_{11}^{\beta_{12}-c} x_{12}^{\beta_{11}+c} x_{21}^{\beta_{22}-d} x_{22}^{\beta_{21}+d} \text {, all } \beta \text {. }
$$

Moreover, $\phi_{q}(\lambda)$ is uniquely determined (up to scalar), by requirement (2.1). Proof. By Frobenius reciprocity [6, I.3.4] we know that for any $G_{\mathbf{Z}}$ module $V$,

$$
\operatorname{Hom}_{G_{z .}}\left(V, \operatorname{ind}_{T_{z}}^{G_{z}}(2 q-2-\lambda)\right) \cong \operatorname{Hom}_{T_{z}}(V, 2 q-2-\lambda),
$$


where the isomorphism associates to any $\psi \in \operatorname{Hom}_{T_{\mathbf{Z}}}(V, 2 q-2-\lambda)$ the mapping $\tilde{\psi} \in \operatorname{Hom}_{G_{\mathbf{z}}}\left(V, \operatorname{ind}_{T_{\mathbf{z}}}^{G_{\mathbf{z}}}(2 q-2-\lambda)\right)$ by

$$
\tilde{\psi}(x)(g)=\left(\psi \otimes \mathrm{id}_{A}\right)\left(g^{-1} \cdot(x \otimes 1)\right),
$$

for all $x \in V, g \in \mathrm{SL}_{2}(A)$, all $A$.

Hence we only need to find the right vector in $\operatorname{ind}_{T_{z}}^{G_{z}} \lambda$ of weight $2 q-2-$ $\lambda$. There are many such vectors in $\operatorname{ind}_{T_{\mathrm{z}}}^{G_{\mathrm{z}}} \lambda$, but only one (up to scalar) in $H_{\mathbf{Z}}^{0}(\lambda)$; it is the vector $v_{\lambda-(q-1)}=x_{12}^{\lambda-(q-1)} x_{22}^{q-1}$. So let $\psi$ be the mapping in $\operatorname{Hom}_{T_{\mathbf{Z}}}\left(\operatorname{ind}_{T_{\mathbf{Z}}}^{G_{\mathbf{Z}}} \lambda, 2 q-2-\lambda\right)$ which maps $v_{\lambda-(q-1)}$ onto 1 and which maps all other basis monomials to zero. (Recall that a basis of monomials for $\operatorname{ind}_{T_{\mathrm{z}}}^{G_{\mathrm{z}}} \lambda$ consists of all $x^{\beta}$ for which $-\lambda=\left(\beta_{11}+\beta_{21}\right)-\left(\beta_{12}+\beta_{22}\right)$ and $\beta_{21} \beta_{12}=0$.)

We need to compute the effect of $\psi$ on an arbitrary monomial $x^{\beta} \in \operatorname{ind}_{T_{\mathbf{Z}}}^{G_{\mathrm{Z}}} \lambda$, taking into account the relation (1.1). It is clear that $\psi\left(x^{\beta}\right)=0$ unless the vector $v_{\lambda-(q-1)}$ is present with nonzero coefficient when $x^{\beta}$ is written in terms of a linear combination of basis vectors. The only $x^{\beta} \in \operatorname{ind}_{T_{z}}^{G_{z}} \lambda$ for which that can happen are those of the form

$$
x^{\beta}=x_{12}^{\lambda-(q-1)+i} x_{21}^{i} x_{22}^{q-1} \quad \text { for some } i \geq 0 .
$$

By (1.1) we have for such an $x^{\beta}$ that

$$
x^{\beta}=\sum_{j=0}^{i}(-1)^{i-j}\left(\begin{array}{l}
i \\
j
\end{array}\right) x_{11}^{j} x_{12}^{i-(q-1)} x_{22}^{(q-1)+j},
$$

and consequently that $\psi\left(x^{\beta}\right)=(-1)^{i}$ for all $x^{\beta}$ of the form (2.5), for all $i \geq 0$.

Now let $x=x^{\beta} \in \operatorname{ind}_{T_{z}}^{G_{z}} \lambda$. Then $g^{-1} \cdot(x \otimes 1)$ is given by (1.3). The terms of (1.3) which will yield something nonzero when $\psi \otimes \mathrm{id}_{A}$ is applied are those terms arising from partitions $\beta=\beta(1)+\beta(2)$ where

$$
\begin{array}{ll}
C_{1} \beta(1)=0, & C_{2} \beta(1)=\lambda-(q-1)+i, \\
C_{1} \beta(2)=i, & C_{2} \beta(2)=q-1,
\end{array}
$$

for $i \geq 0$. Set $c=\beta_{12}(2), d=\beta_{22}(2)$. Then $c+d=q-1$ and $\beta_{12}(1)=$ $\beta_{12}-c, \beta_{22}(1)=\beta_{22}-d$, and

$$
\begin{array}{ll}
R_{1} \beta(1)=\beta_{12}-c, & R_{2} \beta(1)=\beta_{22}-d, \\
R_{1} \beta(2)=\beta_{11}+c, & R_{2} \beta(2)=\beta_{21}+d .
\end{array}
$$

Hence from (2.4), (1.3), and the above we conclude that

$$
\tilde{\psi}\left(x^{\beta}\right)(g)=(-1)^{\beta_{11}+\beta_{21}} \sum_{c+d=q-1}\left(\begin{array}{c}
\beta_{12} \\
c
\end{array}\right)\left(\begin{array}{c}
\beta_{22} \\
d
\end{array}\right) g_{11}^{\beta_{12}-c} g_{12}^{\beta_{11}+c} g_{21}^{\beta_{22}-d} g_{22}^{\beta_{21}+d}
$$


for all $g \in \mathrm{SL}_{2}(A)$ and all $A$, and formula (2.3) follows.

It remains to show (2.2). Recalling that $v_{i}=x_{12}^{i} x_{22}^{\lambda-i}$ by (1.4) we have from (2.3) that

$$
\phi_{q}(\lambda)\left(v_{i}\right)=\sum_{c+d=q-1}\left(\begin{array}{c}
i \\
c
\end{array}\right)\left(\begin{array}{c}
\lambda-i \\
d
\end{array}\right) x_{11}^{i-c} x_{12}^{c} x_{21}^{\lambda-i-d} x_{22}^{d}
$$

for all $i \quad(0 \leq i \leq \lambda)$. Replacing $i-c$ by $a, c$ by $b$, we see that $\lambda-i-d=$ $\lambda-(q-1)-a$ and $d=q-1-b$, so (2.10) becomes

$$
\begin{aligned}
\phi_{q}(\lambda)\left(v_{i}\right) & =\sum_{a+b=i}\left(\begin{array}{l}
i \\
a
\end{array}\right)\left(\begin{array}{c}
\lambda-i \\
q-1-b
\end{array}\right) x_{11}^{a} x_{12}^{b} x_{21}^{\lambda-(q-1)-a} x_{22}^{q-1-b} \\
& =\frac{\left(\begin{array}{c}
\lambda \\
q-1
\end{array}\right)}{\left(\begin{array}{c}
\lambda \\
i
\end{array}\right)} \sum_{a+b=i}\left(\begin{array}{c}
\lambda-(q-1) \\
a
\end{array}\right)\left(\begin{array}{c}
q-1 \\
b
\end{array}\right) x_{11}^{a} x_{12}^{b} x_{21}^{\lambda-(q-1)-a} x_{22}^{q-1-b}
\end{aligned}
$$

and formula (2.2) follows by comparing this with (1.5). The lemma is proved.

Lemma 7. Suppose $\lambda \geq q-1$. There is a $G_{\mathrm{Z}}$ homomorphism

$$
\tilde{\phi}_{q}(\lambda): \operatorname{ind}_{T_{\mathbf{z}}}^{G_{\mathbf{z}}}(2 q-2-\lambda) \rightarrow \operatorname{ind}_{T_{\mathbf{z}}}^{G_{\mathbf{z}}} \lambda
$$

such that

$$
\begin{gathered}
\tilde{\phi}_{q}(\lambda) \text { is nonzero when restricted to } H_{\mathbf{Z}}^{1}(-\lambda-2), \\
\tilde{\phi}_{q}(\lambda)\left(v_{i}^{\prime}\right)=(-1)^{\lambda-(q-1)}\left(\begin{array}{c}
\lambda \\
i
\end{array}\right) v_{i}, \quad \text { all } i(0 \leq i \leq \lambda),
\end{gathered}
$$

$\tilde{\phi}_{q}(\lambda)\left(x^{\beta}\right)$ is given by exactly the same formula as was $\phi_{q}(\lambda)\left(x^{\beta}\right)$, for all $\beta$.

Moreover, $\tilde{\phi}_{q}(\lambda)$ is uniquely determined ( up to scalar) by requirement (2.11). Proof. The uniqueness and the proof of (2.13) are very similar to the proof of the corresponding parts of Lemma 6, so we omit the details. Assuming (2.13), let us prove (2.12).

As $\tilde{\phi}_{q}(\lambda)$ is a $G_{Z}$ homomorphism, it must preserve the action of the Kostant Z-form $\operatorname{Dist}\left(G_{\mathbf{Z}}\right)$. By the remark following the proof of Lemma 3 we have, for 
all $i \quad(0 \leq i \leq \lambda)$, that

$$
\begin{aligned}
\tilde{\phi}_{q}(\lambda)\left(v_{i}^{\prime}\right) & =\tilde{\phi}_{q}(\lambda)\left(X_{-\alpha, i} \cdot v_{0}^{\prime}\right) \\
& =(-1)^{i} X_{-\alpha, i} \tilde{\phi}_{q}(\lambda)\left(v_{0}^{\prime}\right) \\
& =(-1)^{i} X_{-\alpha, i} \tilde{\phi}_{q}(\lambda)\left(x_{21}^{i-(q-1)} x_{22}^{q-1}\right) \\
& =(-1)^{i} X_{-\alpha, i}(-1)^{\lambda-(q-1)} x_{22}^{\lambda} \\
& =(-1)^{\lambda-(q-1)}\left(\begin{array}{c}
\lambda \\
i
\end{array}\right) x_{12}^{i} x_{22}^{\lambda-i} \\
& =(-1)^{\lambda-(q-1)}\left(\begin{array}{c}
\lambda \\
i
\end{array}\right) v_{i} .
\end{aligned}
$$

The proof of the lemma is complete.

Remark. Of course we could have used an argument like this for the proof of (2.2). We gave the other argument to reveal how certain combinatorial identities are implicit in these statements.

We now have constructed homomorphisms of $G_{\mathbf{Z}}$ modules

$$
\begin{aligned}
& \phi_{q}(\lambda): \operatorname{ind}_{T_{\mathbf{z}}}^{G_{\mathbf{z}}} \lambda \rightarrow \operatorname{ind}_{T_{\mathbf{z}}}^{G_{\mathbf{z}}}(2 q-2-\lambda), \\
& \tilde{\phi}_{q}(\lambda): \operatorname{ind}_{T_{\mathbf{z}}}^{G_{\mathbf{z}}}(2 q-2-\lambda) \rightarrow \operatorname{ind}_{T_{\mathbf{z}}}^{G_{\mathbf{z}}} \lambda
\end{aligned}
$$

for all $\lambda \in X\left(T_{\mathrm{Z}}\right)$ such that $\lambda \geq q-1$. If $\lambda \leq q-1$, then $2 q-2-\lambda \geq q-1$ and $\lambda=2 q-2-(2 q-2-\lambda)$, so we see that $\phi_{q}(\lambda)$ can be extended to all $\lambda \in X\left(T_{\mathbf{Z}}\right)$ by setting

$$
\phi_{q}(\lambda)=\tilde{\phi}_{q}(2 q-2-\lambda) \quad \text { for } \lambda<q-1 .
$$

We summarize these results in

Proposition 2. For each positive integer $q$ there is a map $\phi_{q} \in \operatorname{End}_{G_{\mathbf{Z}}}\left(\mathbf{Z}\left[G_{\mathbf{Z}}\right]\right)$ such that $\phi_{q}\left(x^{\beta}\right)$ is given by (2.3) for all $x^{\beta} \in \mathbf{Z}\left[G_{\mathbf{Z}}\right]$. $\phi_{q}$ takes $\mathbf{Z}\left[G_{\mathbf{Z}}\right]_{-\lambda}$ to $\mathbf{Z}\left[G_{\mathbf{Z}}\right]_{\lambda-(2 q-2)}$, for all $\lambda \in X\left(T_{\mathbf{Z}}\right)$. (Recall these are weight spaces under the right regular representation.) Moreover, when $q=1$, the map $\phi_{1}$ is a $G_{\mathbf{Z}}$ module isomorphism between $\mathbf{Z}\left[G_{\mathbf{Z}}\right]_{-\lambda}$ and $\mathbf{Z}\left[G_{\mathbf{Z}}\right]_{\lambda}$, for all $\lambda . \phi_{1}$ is given by

$$
\phi_{1}\left(x^{\beta}\right)=(-1)^{\beta_{11}+\beta_{21}} x_{11}^{\beta_{12}} x_{12}^{\beta_{11}} x_{21}^{\beta_{22}} x_{22}^{\beta_{21}} \text {. }
$$

Finally, for any positive integer $q$, we have $\phi_{q}=\psi_{q} \circ \phi_{1}$, where $\psi_{q}$ is the $G_{\mathrm{Z}}$ endomorphism of $\mathbf{Z}\left[G_{\mathbf{Z}}\right]$ given by

$$
\psi_{q}=\sum_{c+d=q-1}\left(x_{11} \frac{\partial}{\partial x_{12}}\right)^{c}\left(x_{21} \frac{\partial}{\partial x_{22}}\right)^{d} .
$$

Proof. Set $\phi_{q}=\bigoplus_{\lambda \in X\left(T_{\mathrm{Z}}\right)} \phi_{q}(\lambda)$. Check that $\phi_{1} \circ \phi_{1}$ is the identity map. The rest follows by formula (2.3) and the above.

Remark. We have attempted without success to check directly that $\psi_{q}$, as defined by formula $(2.16)$, is a $G_{Z}$ map, without reference to the map $\phi_{q}$. 


\section{Filtrations}

Let $0 \leq \lambda \in X\left(T_{\mathrm{Z}}\right)$. Throughout this section $p$ is a fixed prime. Let $r$ be a nonnegative integer such that $p^{r}-1 \leq \lambda$. Set $q=p^{r}$. We define maps

$$
\begin{aligned}
& T_{r}(\lambda): \operatorname{ind}_{T_{\mathbf{z}}}^{G_{\mathrm{z}}} \lambda \rightarrow \operatorname{ind}_{T_{\mathbf{z}}}^{G_{\mathbf{z}}}(2 q-2-\lambda), \\
& \widetilde{T}_{r}(\lambda): \operatorname{ind}_{T_{\mathbf{z}}}^{G_{\mathbf{z}}}(2 q-2-\lambda) \rightarrow \operatorname{ind}_{T_{\mathbf{z}}}^{G_{\mathbf{z}}} \lambda
\end{aligned}
$$

by

$$
\begin{aligned}
& T_{r}(\lambda)=d^{-1}(\lambda-(q-1)) !(q-1) ! \phi_{q}(\lambda), \\
& \widetilde{T}_{r}(\lambda)=(-1)^{\lambda-(q-1)} \tilde{\phi}_{q}(\lambda),
\end{aligned}
$$

where $d$ is the greatest common divisor of all $i !(\lambda-i) !(0 \leq i \leq \lambda)$. The reason for dividing by $d$ is to be consistent with the approach used by Andersen [1, $\S 3$, cf. [6, II.8.19, Remark 2)]. Clearly $T_{r}(\lambda), \widetilde{T}_{r}(\lambda)$ are homomorphisms of $G_{\mathbf{Z}}$ modules. Recall the bases $\left\{v_{i}\right\},\left\{v_{i}^{\prime}\right\} \quad(0 \leq i \leq \lambda)$ of $H_{\mathbf{Z}}^{0}(\lambda)$ and $H_{\mathbf{Z}}^{1}(-\lambda-2)$, respectively, defined in (1.4), (1.5). By Equations (2.2), (2.12) and the above we have

$$
\begin{aligned}
& T_{r}(\lambda)\left(v_{i}\right)=d^{-1} i !(\lambda-i) ! v_{i}^{\prime}, \\
& \widetilde{T}_{r}(\lambda)\left(v_{i}^{\prime}\right)=\left(\begin{array}{c}
\lambda \\
i
\end{array}\right) v_{i} .
\end{aligned}
$$

Following Andersen, we obtain filtrations of $G_{\mathbf{Z}}$ modules

$$
\operatorname{ind}_{T_{\mathbf{z}}}^{G_{\mathbf{z}}} \lambda=\left(\operatorname{ind}_{T_{\mathbf{z}}}^{G_{\mathbf{z}}} \lambda\right)^{0} \supset\left(\operatorname{ind}_{T_{\mathbf{z}}}^{G_{\mathbf{z}}} \lambda\right)^{1} \supset \cdots
$$

and

$$
\operatorname{ind}_{T_{\mathbf{z}}}^{G_{\mathbf{Z}}}(2 q-2-\lambda)=\left(\operatorname{ind}_{T_{\mathbf{z}}}^{G_{\mathbf{Z}}}(2 q-2-\lambda)\right)^{0} \supset\left(\operatorname{ind}_{T_{\mathbf{Z}}}^{G_{\mathbf{Z}}}(2 q-2-\lambda)\right)^{1} \supset \cdots
$$

defined by

$$
\begin{array}{r}
\left(\operatorname{ind}_{T_{\mathbf{z}}}^{G_{\mathbf{z}}} \lambda\right)^{j}=\left\{v \in \operatorname{ind}_{T_{\mathbf{z}}}^{G_{\mathbf{z}}} \lambda: T_{r}(\lambda)(v) \in p^{j} \operatorname{ind}_{T_{\mathbf{z}}}^{G_{\mathbf{z}}}(2 q-2-\lambda)\right\}, \\
\left.\operatorname{ind}_{T_{\mathbf{z}}}^{G_{\mathbf{z}}}(2 q-2-\lambda)\right)^{j}=\left\{v \in \operatorname{ind}_{T_{\mathbf{z}}}^{G_{\mathbf{z}}}(2 q-2-\lambda): \widetilde{T}_{r}(\lambda)(v) \in p^{j} \operatorname{ind}_{T_{\mathbf{z}}}^{G_{\mathbf{z}}} \lambda\right\} .
\end{array}
$$

The following is easily seen by comparing our setup with [1, §3].

Proposition 3. Let $r$ be such that $p^{r}-1 \leq \lambda<p^{r+1}-1$. Then the above filtrations on $\operatorname{ind}_{T_{\mathbf{z}}}^{G_{\mathbf{z}}} \lambda$, resp., ind $T_{T_{\mathbf{z}}}^{G_{\mathbf{z}}}(2 q-2-\lambda)$ coincide with Andersen's filtrations [1] upon restriction to $H_{\mathrm{Z}}^{0}(\lambda)$, resp., $H_{\mathrm{Z}}^{1}(-\lambda-2)$, after localization at $p$.

Proof. By Proposition 1, Lemmas 6, 7, and the above, the filtration level of each basis vector $v_{i}$ in $H_{\mathrm{Z}}^{0}(\lambda)$ and in $\operatorname{ind}_{T_{\mathbf{Z}}}^{G_{\mathbf{Z}}} \lambda$ is the same, as it is for $v_{i}^{\prime}$ in both $H_{\mathbf{Z}}^{1}(-\lambda-2)$ and $\operatorname{ind}_{T_{\mathbf{Z}}}^{G_{\mathbf{Z}}}(2 q-2-\lambda)$.

Remark. The restriction on $r$ in the above is necessary, as one can see by looking at the case $p=3, \lambda=3, r=0$. 
For any field $k$ let $T=\left(T_{\mathbf{Z}}\right)_{k}$ and $G=\left(G_{\mathbf{Z}}\right)_{k}$ be the groups obtained by the usual extension of scalars. We identify $X\left(T_{\mathrm{Z}}\right)$ with $X(T)$. As $R^{i} \operatorname{ind}_{T_{\mathrm{Z}}}^{G_{\mathrm{Z}}}=0$ for all $i>0$ (see the remark in [6, I.3.3]), by the universal coefficient theorem [1, Th. 1.18] we have, for all $\lambda \in X(T)$,

$$
\left(\operatorname{ind}_{T_{\mathbf{z}}}^{G_{\mathbf{z}}} \lambda\right) \otimes k \cong \operatorname{ind}_{T}^{G} \lambda .
$$

Assume now that $k$ is a field of characteristic $p$. We get filtrations of $\operatorname{ind}_{T}^{G} \lambda$ and $\operatorname{ind}_{T}^{G}(2 q-2-\lambda)$ defined by

$$
\begin{aligned}
\left.\operatorname{(ind}_{T}^{G} \lambda\right)^{j}= & \text { image of }\left(\operatorname{ind}_{T_{\mathbf{z}}}^{G_{\mathbf{z}}} \lambda\right)^{j} \text { under the natural map } \\
& \operatorname{ind}_{T_{\mathbf{z}}}^{G_{\mathbf{Z}}} \lambda \rightarrow\left(\operatorname{ind}_{T_{\mathbf{z}}}^{G_{\mathbf{z}}} \lambda\right) \otimes k,
\end{aligned}
$$

$$
\begin{aligned}
\left.\operatorname{ind}_{T}^{G}(2 q-2-\lambda)\right)^{j}= & \text { image of }\left(\operatorname{ind}_{T_{\mathbf{z}}}^{G_{\mathbf{z}}}(2 q-2-\lambda)\right)^{j} \text { under the natural map } \\
& \operatorname{ind}_{T_{\mathbf{z}}}^{G_{\mathbf{z}}}(2 q-2-\lambda) \rightarrow\left(\operatorname{ind}_{T_{\mathbf{z}}}^{G_{\mathbf{z}}}(2 q-2-\lambda)\right) \otimes k
\end{aligned}
$$

We have embeddings $H^{0}(\lambda) \cong H_{\mathrm{Z}}^{0}(\lambda) \otimes k \rightarrow \operatorname{ind}_{T}^{G} \lambda$ and $H^{1}(-\lambda-2) \cong$ $H_{\mathbf{Z}}^{1}(-\lambda-2) \otimes k \rightarrow \operatorname{ind}_{T}^{G}(2 q-2-\lambda)$. So, by restriction, we get filtrations on $H^{0}(\lambda), H^{1}(-\lambda-2)=H^{1}\left(s_{\alpha} \cdot \lambda\right)$. It is clear that these restricted filtrations coincide with the filtrations constructed in [1]. (Andersen localizes at $p$ before constructing his filtrations, but, as Jantzen points out in [6, II.8.18], that makes no difference.) In particular, the induced filtration on the Weyl module $H^{1} \times$ $(-\lambda-2)$ coincides with the filtration constructed by Jantzen [4] via a contravariant form.

\section{REFERENCES}

1. H. H. Andersen, Filtrations of cohomology modules for Chevalley groups, Ann. Scient. Éc. Norm. Sup. (4) 16 (1983), 495-528.

2. Proc. Amer. Math. Soc. 96 (1986), 537-544.

3. S. R. Doty, Filtrations of rational modules for reductive groups, preprint.

4. J. C. Jantzen, Darstellungen halbeinfacher Gruppen und kontravariante Formen, J. Reine Angew. Math. 290 (1977), 117-141.

5. __ Weyl modules for groups of Lie type, Finite Simple Groups II, (M. Collins, ed.), Academic Press, London and New York, 1980, pp. 291-300.

6. __ Representations of algebraic groups, Academic Press, Orlando and London, 1987.

7. G. Lusztig, Some problems in the representation theory of finite Chevalley groups, Proc. Symp. Pure Math. 37 (1980), 313-317.

Loyola University of Chicago, 6525 North Sheridan, Chicago, Illinois 60626 